\title{
Comparison of Plasma Glucose and Gut Hormone Levels Between Drinking Enteral Formula Over a Period of 5 and 20 Minutes in Japanese Patients With Type 2 Diabetes: A Pilot Study
}

\author{
Kazunari Kamiko a, Kazutaka Aoki ${ }^{\text {a, b, d }}$, Hiroshi Kamiyama ${ }^{\text {a }}$, Masataka Taguric, \\ Yasuo Terauchi ${ }^{\mathrm{a}, \mathrm{d}}$
}

\begin{abstract}
Background: A fast eating speed is reportedly associated with obesity, fatty liver, and metabolic syndrome. As a comparison of postprandial glucose levels after eating quickly or slowly has not been previously reported for Japanese patients with type 2 diabetes, we evaluated the impact of the fast or slow ingestion of an enteral formula (liquid meal) on glucose metabolism.
\end{abstract}

Methods: Ten Japanese patients with type 2 diabetes who had been hospitalized at our hospital were enrolled. All the subjects received an enteral formula for breakfast. The study was performed over a 2-day period in each subject (day 1: enteral formula was consumed over a 5-minute period; day 2: enteral formula was consumed over a 20-minute period). The subjects were requested to fast for at least 12 hours before eating breakfast, and blood samples were collected at 0 , 30,60 , and $120 \mathrm{~min}$ after the start of breakfast.

Results: The areas under the curve (AUCs) of the plasma glucose, serum insulin, plasma active ghrelin, glucagon-like peptide-1 (GLP-1), plasma total glucose-dependent insulinotropic polypeptide (GIP), and serum total peptide YY (PYY) levels were not significantly changed by intake over a 5 -minute or 20-minute period.

Conclusions: Eating quickly per se probably does not affect postprandial glucose excursions, but the increased energy intake resulting from eating quickly may increase the body weight and increase insulin resistance. Eating quickly may increase energy intake and worsen long-term metabolic parameters.

Manuscript accepted for publication August 08, 2016

aDepartment of Endocrinology and Metabolism, Yokohama City University Graduate School of Medicine, Japan

bInternal Medicine, Kanagawa Dental University, Japan

'Department of Biostatistics, Yokohama City University Graduate School of Medicine, Japan

${ }^{\mathrm{d} C}$ Corresponding Author: Kazutaka Aoki, Yasuo Terauchi, Department of Endocrinology and Metabolism, Yokohama City University Graduate School of Medicine, Fuku-ura 3-9, Kanazawa-ku, Yokohama 236-0004, Japan.

Email:k.aoki@kdu.ac.jp, terauchi@yokohama-cu.ac.jp

doi: http://dx.doi.org/10.14740/jocmr2686w
Keywords: Eating rate; Enteral formula; Type 2 diabetes; Blood glucose; Incretin

\section{Introduction}

Diet therapy is crucial for treating patients with type 2 diabetes and for preventing diabetes complications. Eating slowly is commonly recognized as being important, and eating quickly is reportedly associated with obesity, fatty liver, and metabolic syndrome [1-6]. In a cross-sectional study, eating quickly was associated with the body mass index (BMI), but eating speed was not associated with the HbAlc level in Japanese patients with type 2 diabetes [7]. In another cross-sectional study, eating quickly was found to be a possible risk factor for the development of diabetes in Japanese subjects [8]. Requesting obese adolescents to eat more slowly for 12 months decreased the BMI, HOMA-IR, and plasma ghrelin levels and increased the peptide YY (PYY) levels [9]. Only one report, however, has directly evaluated the effects of eating speed on postprandial glucose levels and gut hormones [10]. Thus, in overweight or obese patients with type 2 diabetes, eating slowly did not change the postprandial glucose, insulin, PYY, glucagon-like peptide 1 (GLP-1), or ghrelin levels, but it was correlated with increased fullness and decreased hunger ratings, compared with eating quickly [10].

However, since the postprandial glucose levels after eating quickly or slowly have not been previously compared in Japanese patients with type 2 diabetes, we evaluated the impact of quickly or slowly ingesting an enteral formula (a liquid meal) on glucose metabolism.

\section{Materials and Methods}

We conducted the present study with the approval of the Institutional Ethics Review Committee of the Yokohama City University Hospital; the UMIN Clinical Trial Registry number was 000017632. Informed consent was obtained from each subject before the start of the study. Ten Japanese patients with type 2 diabetes who had been hospitalized in our hospital were enrolled. 

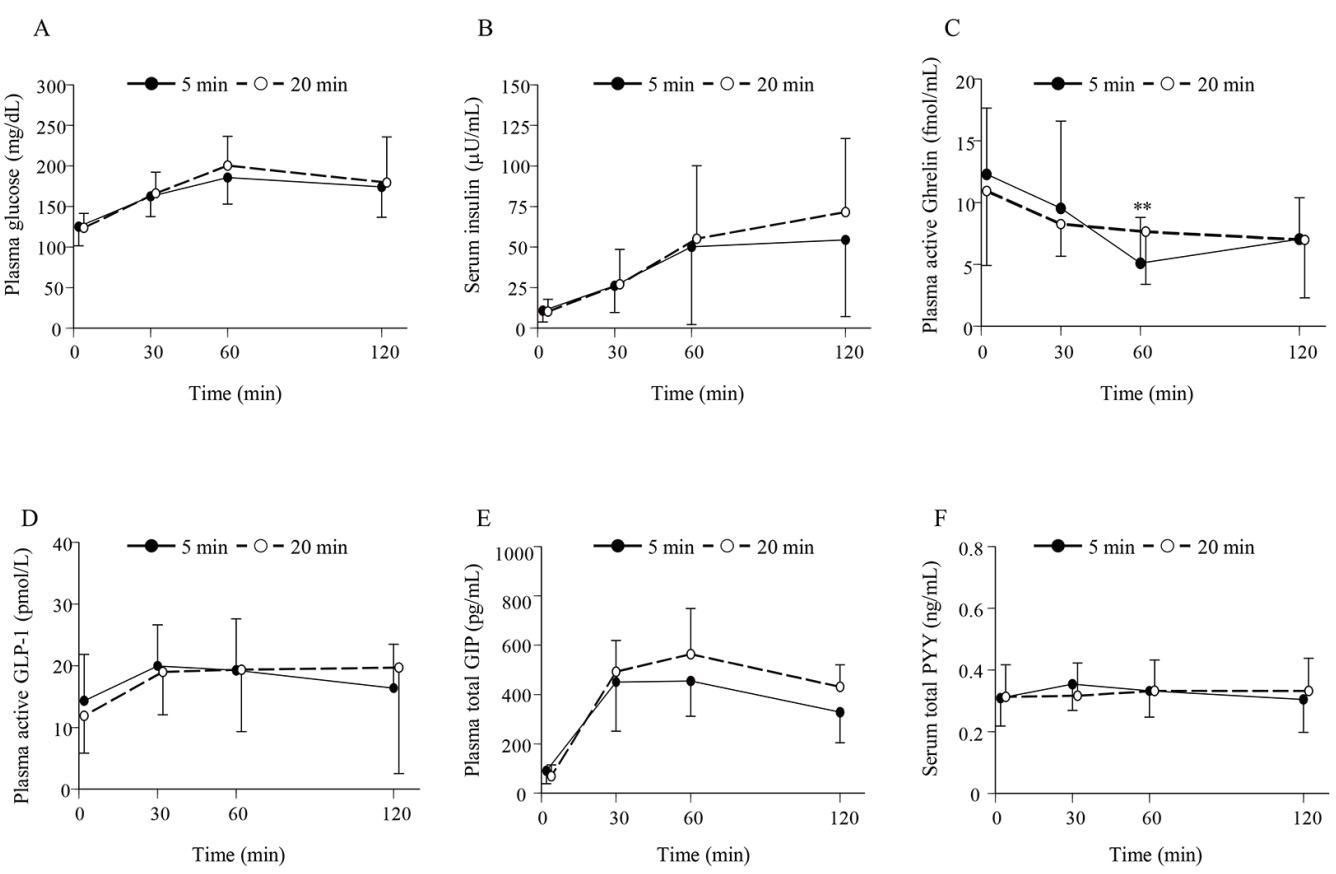

Figure 1. (A) Time profiles for the plasma glucose, (B) serum insulin, (C) plasma active ghrelin, (D) plasma active GLP-1, (E) plasma total GIP, and $(F)$ serum total PYY levels for the 5-min and 20-min groups. Data are expressed as the mean \pm SD. ${ }^{*} P<$ 0.01 between two groups.

The patients who had been treated with diet therapy or with oral hypoglycemic agents (except for DPP-4 inhibitors and alpha glucosidase inhibitors) were enrolled. This study was carried out under a stable glycemic condition in the patients, and the patients' medication prescription was not changed during the study. All the subjects received an enteral formula (Sanet-2.0 ${ }^{\circledR}$, $400 \mathrm{kcal}$; protein: $16 \mathrm{~g}$; fat: $15 \mathrm{~g}$; carbohydrate: $49.2 \mathrm{~g}$ ) for breakfast. This trial was performed over a 2-day period in each subject (day 1: Sanet-2.0 ${ }^{\circledR}$ was consumed over a 5-min period; day 2: Sanet-2.0 ${ }^{\circledR}$ was consumed over a 20 -minute period). For the study, the subjects were requested to fast for at least $12 \mathrm{~h}$ before eating breakfast. Blood samples were collected at 0,30 , 60 , and $120 \mathrm{~min}$ after the start of breakfast. Hydrochloride was added to one blood sample from each subject to measure the active ghrelin level. One blood sample was withdrawn from the catheter directly into a BD P700 Blood Collection Tube (catalogue no. 366473; BD, Franklin Lakes, NJ, USA) containing a DPP-4 inhibitor to measure the plasma active GLP-1 levels. The plasma glucose and serum insulin levels were measured by Hokenkagaku Institute Inc. (Yokohama, Japan). Active ghrelin was measured using an ELISA kit (Sceti, Tokyo, Japan). Plasma active GLP-1 and GIP were measured using an ELISA kit (Immuno-biological Laboratories Co., Ltd, Fujioka, Japan, and Millipore Corporation, MA, USA, respectively). The serum total PYY level (PYY1-36 and PYY3-36) was measured using an EIA kit (Yanaihara Institute Inc., Shizuoka, Japan).

Data were expressed as the mean $\pm \mathrm{SD}$. The areas under the curve (AUCs) from just before the meal until $120 \mathrm{~min}$ after the start of the meal were calculated using the trapezoid method. The analyses for the time courses and the AUCs were performed using a paired $t$-test. The statistical analyses were conducted using Ekuserutoukei 2012 software (Social Survey Research Information Co., Ltd, Tokyo, Japan).

\section{Results}

Ten patients (seven men and three women) with a mean age of $57.4 \pm 15.1$, a mean body height of $164.9 \pm 7.8 \mathrm{~cm}$, a body weight of $70.6 \pm 17.3 \mathrm{~kg}$, a BMI of $25.6 \pm 4.4 \mathrm{~kg} / \mathrm{m}^{2}$, and a mean HbAlc of $10.2 \pm 1.9 \%$ were enrolled in the present study. The duration of diabetes was $7 \pm 8$ years. One patient had been treated with dietary therapy alone (drug-naive), four patients had been treated with sulphonylurea alone, three patients had been treated with metformin alone, and two patients had been treated with sulphonylurea and metformin.

As shown in Figure 1A, no significant difference in the time course for the plasma glucose level was seen between the consumption of the enteral formula over a 5 -min period (5-min group) and over a 20-min period (20-min group). Consequently, no significant difference in the AUCs for plasma glucose was seen between the 5-min and the 20-min groups (Fig. 2A). Similarly, no significant differences were seen in the time courses for the serum insulin, plasma active GLP-1, total GIP, and total PYY levels between the 5-min and the 20min groups (Fig. 1B, D, E, F). Therefore, no significant differences in the AUCs for serum insulin, plasma active GLP-1, total GIP, or total PYY were observed between the 5-min and the 20-min groups (Fig. 2B, D, E, F). As for the time course for plasma ghrelin, its level at $60 \mathrm{~min}$ after the consumption of the enteral formula was significantly higher in the 5-min group than in the 20 min-group; however, no significant differences 


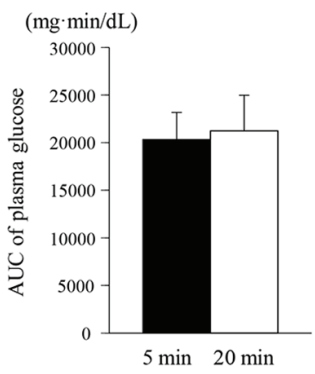

$\mathrm{D}$

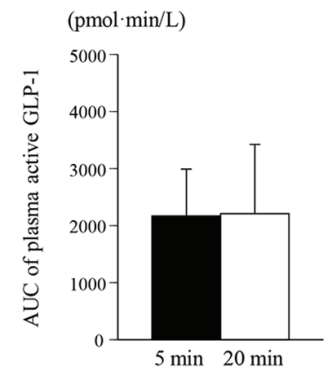

B

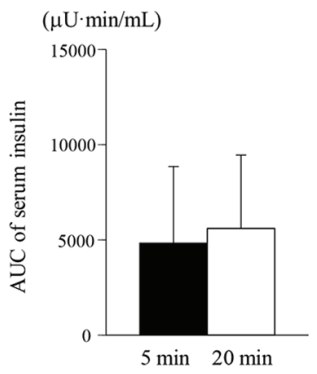

$\mathrm{E}$

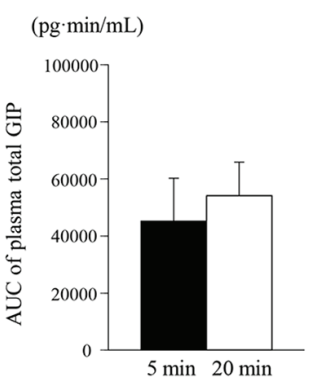

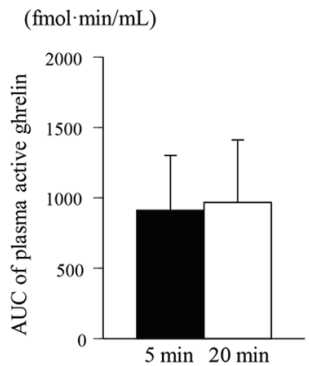

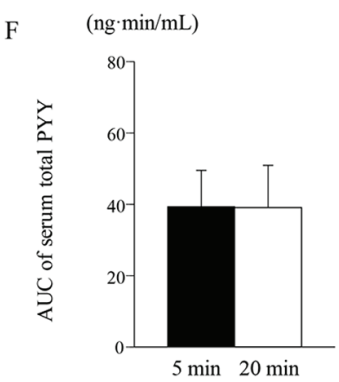

Figure 2. Area under the curve (AUC) for the plasma glucose (A), serum insulin (B), plasma active ghrelin (C), plasma active GLP-1 (D), plasma total GIP (E), and serum total PYY (F) levels from 0 to 120 min for the 5-min and 20-min groups. Data are expressed as the mean \pm SD.

in the plasma ghrelin levels at 0,30, and 120 min were seen between the two groups. Consequently, no significant difference in the AUC for plasma ghrelin was seen between the two groups (Figs. 1C and 2C).

\section{Discussion}

The most important finding of our study was that the AUCs for plasma glucose, serum insulin, plasma active ghrelin, GLP-1, plasma total GIP, and serum total PYY did not differ significantly between the 5-min and the 20-min groups. As GLP-1 and PYY are known to decrease appetite and ghrelin is known to increase appetite, we measured these hormones, including the total GIP; however, no distinct changes were observed after the ingestion of a single liquid meal [11-13]. These results were in accordance with a previous report [10].

The ingestion speed of a single liquid meal did not influence the postprandial glucose level in patients with type 2 diabetes. Eating quickly is associated with obesity [1-4]. Eating slowly has been reported to increase fullness and to decrease the hunger rating, leading to a decreased energy intake [10, 14]. Indeed, the eating speed was associated with the onset of diabetes over a 7-year study period, but the association between eating speed and the onset of diabetes was not significant after adjustment for the BMI [15]. Therefore, eating quickly per se does not affect postprandial glucose excursions, but the increased energy intake resulting from eating quickly may increase the body weight and increase insulin resistance.

In healthy subjects, the AUC for the plasma glucose level after the ingestion of hydrolyzed starch over a 1-min period was smaller than that over a 10 -min period [16]. In healthy subjects, the ingestion of a single meal slowly (22 min) resulted in a significant increase in blood glucose and ghrelin levels, compared with a normal pace of eating (10 min) [17]. As no significant differences in the postprandial glucose level according to ingestion speed were observed in healthy subjects (preliminary data not shown), further examinations in healthy subjects are needed.

In Japan, calculations of the daily energy intake are commonly performed as part of diet therapy for patients with diabetes. The postprandial glucose level did not differ after the ingestion of a single enteral formula either quickly or slowly. However, obese patients with type 2 diabetes should be advised not to increase their energy intakes by eating quickly in their daily lives.

The present study had several limitations. The number of patients was relatively small, and this investigation examined the results after the ingestion of only a single enteral formula. As it is difficult to change an individual's normal eating speed, patients were instructed to consume the enteral formula over a defined period of time. Therefore, a larger-scale study in which the patients are instructed to eat a normal diet, rather than a liquid meal, either slowly or quickly for a longer time period is needed in the future.

\section{Conclusions}

The postprandial plasma glucose, serum insulin, plasma active ghrelin, GLP-1, total GIP, and serum total PYY levels were not significantly changed by the intake of a single enteral formula 
either quickly or slowly. Eating quickly may increase the energy intake and worsen long-term metabolic parameters.

\section{Conflicts of Interest}

Terauchi Y received honoraria for lectures from MSD; Ono Pharmaceutical Co. Ltd; Boehringer Ingelheim GmbH; Takeda Pharmaceutical Company Ltd; Tanabe-Mitsubishi Pharma; Daiichi-Sankyo Company Ltd; Sanwa Kagaku Kenkyusho Co., Ltd; Novo Nordisk Pharma Ltd; Eli Lilly and Company; Sanofi, DaiNippon-Sumitomo, Shionogi \& Co.; Ltd, Bayer Yakuhin Ltd, Astellas Pharma Inc.; and Astra Zeneca and obtained research support from MSD; Ono Pharmaceutical Co. Ltd; Boehringer Ingelheim GmbH; Takeda Pharmaceutical Company Ltd; Tanabe-Mitsubishi Pharma; Daiichi-Sankyo Company Ltd; Sanwa Kagaku Kenkyusho Co., Ltd; Novo Nordisk Pharma Ltd; Eli Lilly and Company; Sanofi, Astellas Pharma Inc.; and Astra Zeneca. Aoki K obtained research support from Sanwa Kagaku Kenkyusho Co., Ltd. Kamiko K, Kamiyama $\mathrm{H}$ and Taguri $\mathrm{M}$ declare that they have no conflict of interest.

\section{References}

1. Ohkuma T, Hirakawa Y, Nakamura U, Kiyohara Y, Kitazono $\mathrm{T}$, Ninomiya $\mathrm{T}$. Association between eating rate and obesity: a systematic review and meta-analysis. Int J Obes (Lond). 2015;39(11):1589-1596.

2. Sasaki S, Katagiri A, Tsuji T, Shimoda T, Amano K. Selfreported rate of eating correlates with body mass index in 18-y-old Japanese women. Int J Obes Relat Metab Disord. $2003 ; 27(11): 1405-1410$.

3. Otsuka R, Tamakoshi K, Yatsuya H, Murata C, Sekiya A, Wada K, Zhang HM, et al. Eating fast leads to obesity: findings based on self-administered questionnaires among middle-aged Japanese men and women. J Epidemiol. 2006;16(3):117-124.

4. Kral JG, Buckley MC, Kissileff HR, Schaffner F. Metabolic correlates of eating behavior in severe obesity. Int $\mathrm{J}$ Obes Relat Metab Disord. 2001;25(2):258-264.

5. Zhu B, Haruyama Y, Muto T, Yamazaki T. Association between eating speed and metabolic syndrome in a three-year population-based cohort study. J Epidemiol. 2015;25(4):332-336.

6. Nagahama S, Kurotani K, Pham NM, Nanri A, Kuwahara K, Dan M, Nishiwaki Y, et al. Self-reported eating rate and metabolic syndrome in Japanese people: cross-sectional study. BMJ Open. 2014;4(9):e005241.

7. Saito A, Kawai K, Yanagisawa M, Yokoyama H, Kuribayashi N, Sugimoto H, Oishi M, et al. Self-reported rate of eating is significantly associated with body mass index in Japanese patients with type 2 diabetes. Japan Diabetes Clinical Data Management Study Group (JDDM26). Appetite. 2012;59(2):252-255.

8. Yamazaki T, Yamori M, Asai K, Nakano-Araki I, Yamaguchi A, Takahashi K, Sekine A, et al. Mastication and risk for diabetes in a Japanese population: a cross-sectional study. PLoS One. 2013;8(6):e64113.

9. Galhardo J, Hunt LP, Lightman SL, Sabin MA, Bergh C, Sodersten P, Shield JP. Normalizing eating behavior reduces body weight and improves gastrointestinal hormonal secretion in obese adolescents. J Clin Endocrinol Metab. 2012;97(2):E193-201.

10. Angelopoulos T, Kokkinos A, Liaskos C, Tentolouris N, Alexiadou K, Miras AD, Mourouzis I, et al. The effect of slow spaced eating on hunger and satiety in overweight and obese patients with type 2 diabetes mellitus. BMJ Open Diabetes Res Care. 2014;2(1):e000013.

11. Turton MD, O'Shea D, Gunn I, Beak SA, Edwards CM, Meeran K, Choi SJ, et al. A role for glucagon-like peptide-1 in the central regulation of feeding. Nature. 1996;379(6560):69-72.

12. Woods SC, D'Alessio DA. Central control of body weight and appetite. J Clin Endocrinol Metab. 2008;93(11 Suppl 1):S37-50.

13. Kojima M, Kangawa K. Ghrelin, an orexigenic signaling molecule from the gastrointestinal tract. Curr Opin Pharmacol. 2002;2(6):665-668.

14. Sakurai M, Nakamura K, Miura K, Takamura T, Yoshita K, Nagasawa SY, Morikawa Y, et al. Self-reported speed of eating and 7-year risk of type 2 diabetes mellitus in middle-aged Japanese men. Metabolism. 2012;61(11):15661571 .

15. Andrade AM, Greene GW, Melanson KJ. Eating slowly led to decreases in energy intake within meals in healthy women. J Am Diet Assoc. 2008;108(7):1186-1191.

16. Heine RJ, Hanning I, Morgan L, Alberti KG. The oral glucose tolerance test (OGTT): effect of rate of ingestion of carbohydrate and different carbohydrate preparations. Diabetes Care. 1983;6(5):441-445.

17. Sobki SH, Zaid AA, Khan HA, Alhomida AS, Hilal KA, Khan SA. Significant impact of pace of eating on serum ghrelin and glucose levels. Clin Biochem. 2010;43(45):522-524. 